\title{
Case Report: Retirees' Acceptance and Perceived Contribution of Smartphone in Chronic Disease Management
}

\author{
Mei-Ju Chen \\ Department of Prevention Medicine, Taipei City Hospital, Department of Family Medicine, Heping Fuyou \\ Branch of Taipei City Hospital, College of Healthcare Administration and Management, \\ National Taipei University of Nursing and Health Science \\ Email: DXD41@tpech.gov.tw
}

Received April 2014

\begin{abstract}
Objectives: As smartphones become more popular, so do their applications. However, expectations of the elderly regarding the contribution of smartphone in controlling chronic diseases remain unclear. This research aims to understand senior retirees' smartphone acceptance, perceived contribution of smartphone application in facilitating chronic disease control and their association. Findings from the study provide insights for the development of mobile applications in chronic disease management. Methods: convenience sampling was conducted to recruit 110 senior retirees who worked as volunteers in a regional hospital in Taipei. Data was collected through a structured questionnaire. Descriptive, chi-square and logistic regression statistics were applied to analyze data. Results: A total of 108 completed questionnaires were collected with a return rate of $98.2 \%$. Mean age was $65.34 \pm 9.59$ years old. 0 all respondents, $40.7 \%$ reported acceptance of internet-enabled smartphones and $54.6 \%$ expected that smartphones would facilitate chronic disease management in the future. However, a statistically significant $37.3 \%$ of those expecting smartphone to play a role in disease management did not accept smartphones yet. After controlling for age and education, logistic regression analysis showed that older adults with higher smartphone acceptance were more likely to expect use of smartphone in case management (OR $=7.439, \mathrm{p}<0.001$ ). Conclusions: The research presented a scope for smartphone application to control chronic disease in the future. Despite a relatively lower level of smartphone acceptance, the elderly still expected a positive role for mobile appliances to play in chronic disease management.
\end{abstract}

\section{Keywords}

Smartphone, Elderly, Chronic Disease Management

\section{Introduction}

Growing numbers of older adults are accompanied with increasing consumption capacity and influence of the 
elderly population. A notable example is higher use of mobile phones. Technological advances also changed the way senior home care is delivered. However, product development and marketing still neglects the need of the elderly, which is different from that of the young generation. Research showed that the elderly were interested in mobile phone use and applications when such services contributed to a more social, active, meaningful and independent life [1].

Research observed that many elderly Americans started to use mobile phones despite various limitations and challenges [2]. Differences existed between older and younger elderly with regards to the motivation for mobile phone use. An exploratory factor analysis identified usefulness and security as two underlying and correlated determinants. Specifically, education, ease of use, usefulness, security and short/long-distance travel frequency were significantly important predictors [3]. A study on digital engagement of elderly people pointed out that mobile phones were mostly used to stay connected with family members and friends.

In some parts of the world, many elderly people are at risk due to geographic and social isolation in combination with certain chronic conditions. Information and communications technology (ICT) has brought new dimensions to the delivery of healthcare. For example, targeting patients with chronic illness, some project has developed services interconnecting mobile platforms and computers [4].

A revolutionary mobile health model has evolved from conventional mode of telemedicine, thanks to the latest advancement of smartphone technology. It allows users to access fast, pervasive, cheap and powerful mobile platforms at one end, and enables health systems to deliver services and information at the other, making a mobile healthcare network possible [5]. The new mode of mobile health has become a high potential solution to address issues from skyrocketing cost, malpractice, manpower shortage, resource constraints, demand for quality, to physician-patient communication [6].

This research aims to understand senior retirees' smartphone acceptance, perceived contribution of smartphone application in facilitating chronic disease control and their association.

\section{Methods}

This cross-sectional research adopted convenience sampling method. It recruited 110 senior retirees who worked as volunteers in a regional hospital in Taipei. The questionnaire obtained personal data including sex, age and education, whether respondents accepted internet-enabled smartphones and whether they agreed future internetenabled smartphones could facilitate chronic disease management. A 5-point Likert scale was used to measure the extent to which a respondent agreed with the two statements, with 5 representing strong agreement (acceptance) and 1 representing strong disagreement (non-acceptance). The Cronbach's $\alpha$ for the scale was 0.72 . Descriptive, chi-square and logistic regression statistics were applied to analyze data.

\section{Results}

Totally 108 questionnaires were completed with a return rate of $98.2 \%$. The majority of volunteers in the hospital were female (more than $90 \%$ ). The mean age of the sample was $65.34 \pm 9.59$ years old. Overall, $40.7 \%$ reported acceptance of internet-enabled smartphones and 54.6\% expected that smartphones would facilitate chronic disease management in the future. As shown in Table 1, among those with higher acceptance of internet-enabled smartphones $45.5 \%$ were high school graduates, $38.6 \%$ were college graduates, $50.0 \%$ were below 60 years of age, $43.2 \%$ between $60-70$, and $58.1 \%$ had chronic diseases. Of those who agreed that internetenabled smartphones could facilitate chronic disease management, $44.1 \%$ were high school graduates, $35.6 \%$ college graduates, $49.2 \%$ between 60 - 70, and 59.6\% with chronic conditions. However, a statistically significant 37.3\% ( $<$ 0.001) of those expecting smartphone to play a role in disease management did not accept smartphones yet. In Table 2, after controlling for age and education, logistic regression analysis found that older adults with higher smartphone acceptance were more likely to expect future application of smartphone in case management $(\mathrm{OR}=7.439, \mathrm{p}<0.001)$.

\section{Discussion}

Narula (1988) [7] noted that implementation of communication technology had a profound impact on culture and the way people interacted with each other in a society. New technology first affected an individual's state of 
Table 1. Demographic characteristics.

\begin{tabular}{|c|c|c|c|c|c|c|c|c|c|c|}
\hline & \multicolumn{5}{|c|}{$\begin{array}{c}\text { Smartphones can facilitate } \\
\text { chronic disease management }(\mathrm{N}=108)\end{array}$} & \multicolumn{5}{|c|}{ Smartphone acceptance $(\mathrm{N}=108)$} \\
\hline & $\begin{array}{l}\text { Unlikely } \\
\text { (N) }\end{array}$ & $\%$ & Likely (N) & $\%$ & $\mathrm{p}$ & $\begin{array}{l}\text { Unlikely } \\
(\mathrm{N})\end{array}$ & $\%$ & $\begin{array}{l}\text { Likely } \\
(\mathrm{N})\end{array}$ & $\%$ & $\mathrm{p}$ \\
\hline Education & & & & & 0.009 & & & & & 0.011 \\
\hline Primary school & 9 & $18.80 \%$ & 5 & $8.50 \%$ & & 12 & $19.00 \%$ & 2 & $4.50 \%$ & \\
\hline Junior high & 15 & $31.30 \%$ & 7 & $11.90 \%$ & & 17 & $27.00 \%$ & 5 & $11.40 \%$ & \\
\hline Senior high & 10 & $20.80 \%$ & 26 & $44.10 \%$ & & 16 & $25.40 \%$ & 20 & $45.50 \%$ & \\
\hline College and above & 14 & $29.20 \%$ & 21 & $35.60 \%$ & & 18 & $28.60 \%$ & 17 & $38.60 \%$ & \\
\hline Sex & & & & & N.P. & & & & & N.P. \\
\hline Male & 5 & $10.20 \%$ & 4 & $6.80 \%$ & & 5 & $7.80 \%$ & 4 & $9.10 \%$ & \\
\hline Female & 44 & $89.80 \%$ & 55 & $93.20 \%$ & & 59 & $92.20 \%$ & 40 & $90.90 \%$ & \\
\hline Age $(65.34 \pm 9.59)$ & & & & & 0.005 & & & & & 0.000 \\
\hline$<60$ & 12 & $24.5 \%$ & 23 & $39.0 \%$ & & 13 & $20.3 \%$ & 22 & $50.0 \%$ & \\
\hline $60-70$ & 17 & $34.7 \%$ & 29 & $49.2 \%$ & & 27 & $42.2 \%$ & 19 & $43.2 \%$ & \\
\hline $70-80$ & 15 & $30.6 \%$ & 4 & $6.8 \%$ & & 18 & $28.1 \%$ & 1 & $2.3 \%$ & \\
\hline$>80$ & 5 & $10.2 \%$ & 3 & $5.1 \%$ & & 6 & $9.4 \%$ & 2 & $4.5 \%$ & \\
\hline Chronic dise & & & & & N.P. & & & & & N.P. \\
\hline No & 20 & $43.50 \%$ & 23 & $40.40 \%$ & & 25 & $41.70 \%$ & 18 & $41.90 \%$ & \\
\hline Yes & 26 & $56.50 \%$ & 34 & $59.60 \%$ & & 35 & $58.30 \%$ & 25 & $58.10 \%$ & \\
\hline \multicolumn{11}{|c|}{ Mobile phone acceptance } \\
\hline Unlikely & 42 & $85.7 \%$ & 22 & $37.3 \%$ & 0.000 & & & & & \\
\hline Likely & 7 & $14.3 \%$ & 37 & $62.7 \%$ & & & & & & \\
\hline
\end{tabular}

Note: ${ }^{*} \mathrm{p}<0.05,{ }^{* *} \mathrm{p}<0.01,{ }^{* * *} \mathrm{p}<0.001$.

Table 2. Logistic regression analysis.

\begin{tabular}{|c|c|c|c|c|}
\hline & \multirow{2}{*}{ OR } & \multicolumn{3}{|c|}{$95 \%$ CI } \\
\hline & & & Lower & Upper \\
\hline $\begin{array}{l}\text { Smartphone } \\
\text { acceptance }\end{array}$ & 7.439 & $* * *$ & 2.707 & 20.442 \\
\hline
\end{tabular}

Note: Controlling for age and education. Low level of mobile phone acceptance as reference group. ${ }^{*} \mathrm{p}<0.05,{ }^{* *} \mathrm{p}<0.01,{ }^{* * *} \mathrm{p}<0.001$.

mind, then the way of thinking and acting, and gradually the sense and perception of human beings (Sprague and Ruud, 1998) [8]. Chang (1999) [9] held that mobile phones, more than just a tool of communication, highlighted the meaning of interpersonal relationships and connectedness, which was highly valued in Chinese culture. Local research found that the elderly adopted mobile phones for easy communication [10]. In this research, $40.7 \%$ of the elderly respondents reported acceptance of internet-enabled smartphones. There was no significant association between having chronic diseases and expecting future mobile phones to facilitate chronic disease control. Consistent with existing research findings [2], those with better education reported higher level acceptance of smartphones.

In the research respondents aged below 60 years showed higher acceptance of internet-enabled smartphones, but those between 60 and 70 years constituted the majority expecting smartphones to facilitate disease management. Other researchers held the view that the elderly generally showed positive attitudes towards the collection 
of health monitoring information, recognizing the utility of these data sources. Some seniors also mentioned increased social interactions generated in the community setting [11]. However, in the current study $37.3 \%$ of the elderly did not accept smartphones despite their expectations for the possible contribution of future smartphones.

Elderly people are usually favorable towards issues they are aware of. On the other hand when facing novelty or unfamiliar issues they demonstrate lessened confidence, memory, reasoning, judgment and even eye-hand coordination due to physical and psychological ageing. A new motion or operation without previous knowledge will create difficulties. As a result, their ability of information processing, logical thinking or postural and spatial coordination deteriorates. This tendency becomes evident when they operate new products. The elderly tend to lose confidence in using new gadgets when they have less control of the devices [12].

Nowadays smartphones perform multiple tasks, but the elderly people prefer that mobile phones as simple as possible because most of them only use phones to make and receive calls. In this sense, mobile phones can be designed to meet the expectations of this particular group. Most seniors are now aware that they can change phones to solve usage barriers such as small display or buttons, but from accepting smartphones to seeing them as a tool to manage disease is an area little studied. The context of the elderly mobile phone users is worth research because it will provide insights in the development of an everyday high-tech, elderly-friendly product.

\section{Discussion}

The research found that the elderly with higher acceptance of smartphones were more likely to expect smartphones to facilitate chronic disease management. While more and more elderly people possess mobile phones, the extent to which mobile phones integrate into daily life differs. The research presented a scope for future smartphone application in chronic disease control. Despite a relatively lower level of smartphone acceptance, the elderly still expected a positive role for mobile appliances to play in chronic disease management. We suggest that future design of mobile phones be informed by the lifestyle and needs of users. We also suggest that new strategy be adopted to make health management an integral part of overall need assessment.

\section{References}

[1] Hardill, I. and Olphert, C.W. (2012) Staying Connected: Exploring Mobile Phone Use amongst Older Adults in the UK. Geoforum, 43, 1306-1312. http://dx.doi.org/10.1016/j.geoforum.2012.03.016

[2] Mallenius, S., Rossi, M. and Tuunainen, V.K. (2007) Factors Affecting the Adoption and Use of Mobile Devices and Services by Elderly People-Results from a Pilot Study. In: Proceedings of 6th Annual Global Mobility Roundtable, Los Angeles.

[3] Kubik, S. (2009) Motivations for Cell Phone Use by Older Americans. Gerontechnology, 8, 150-164. http://dx.doi.org/10.4017/gt.2009.08.03.007.00

[4] Panou, M., Touliou, K., Bekiaris, E. and Tsaprounis, T. (2013) Mobile Phone Application to Support the Elderly. International Journal of Cyber Society and Education, 6, 51-56. http://dx.doi.org/10.7903/ijcse.1047

[5] Varshney, U. (2003) Pervasive Healthcare. Computer, 36, 138-140. http://dx.doi.org/10.1109/MC.2003.1250897

[6] PerCare 2008 (2007) First International Workshop on Pervasive Digital Healthcare.

[7] Narula, U. (1988) The Cultural Challenge of Communication Technology. American Behavioral Scientist, 32, $194-207$. http://dx.doi.org/10.1177/0002764288032002010

[8] Sprague, J. and Ruud, G.L. (1988) Boat-Rocking in the High-Technology Culture. American Behavioral Scientist, 32, 169-193. http://dx.doi.org/10.1177/0002764288032002009

[9] S.S., C. (1999) Why Are Mobile Phones Popular in Taiwan? Commonwealth Magazine, 68-74.

[10] S.Y., L. (2010) An Investigation on the Elderly's Mobile Phone Use and Using Behavior in Northern Taiwan. Department of Industrial Engineering, National Tsing Hua University, Hsinchu City, 51.

[11] Demiris, G., Thompson, H., Boquet, J., Le, T., Chaudhuri, S. and Chung, J. (2013) Older Adults’ Acceptance of a Community-Based Telehealth Wellness System. Inform Health Soc Care, 38, 27-36. http://dx.doi.org/10.3109/17538157.2011.647938

[12] Chang, S.-W. and Lee, K.-K. (2002) Studies of Design Considerations for Human Needs in High Tech Products Using Mobile Handsets for Senior Citizens as an Example. Zao Xing Yi Shu Xue Kan, 357-368. 
Scientific Research Publishing (SCIRP) is one of the largest Open Access journal publishers. It is currently publishing more than 200 open access, online, peer-reviewed journals covering a wide range of academic disciplines. SCIRP serves the worldwide academic communities and contributes to the progress and application of science with its publication.

Other selected journals from SCIRP are listed as below. Submit your manuscript to us via either submit@scirp.org or Online Submission Portal.
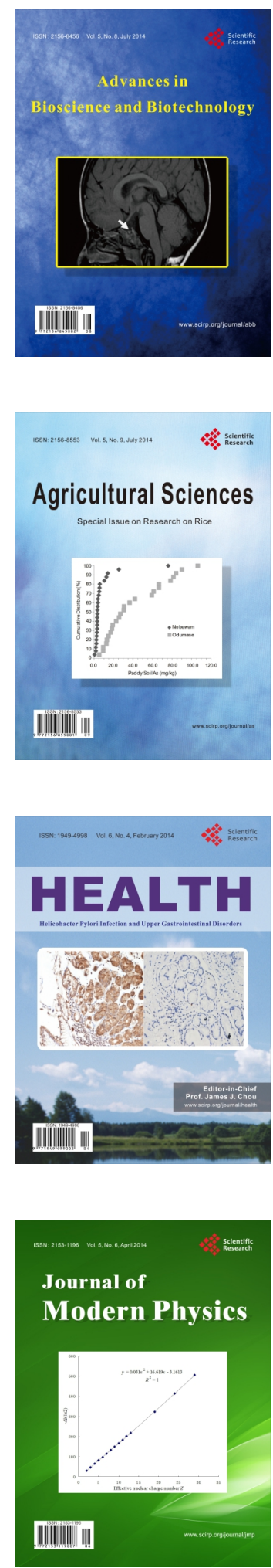
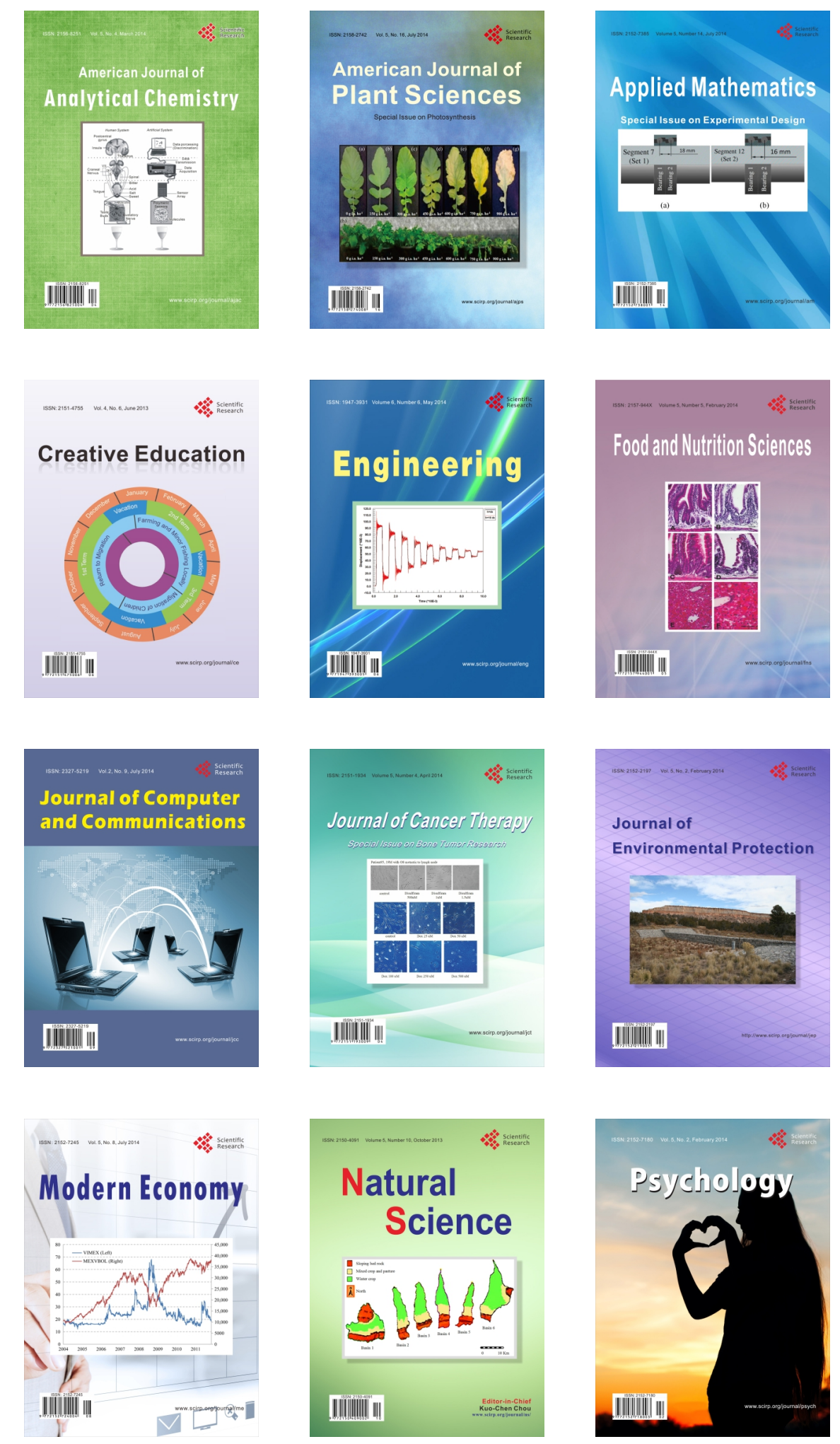